\title{
Numerical Prediction of the Response of Metal-to-Metal Adhesive Joints with Ductile Adhesives
}

\author{
N.G. Tsouvalis ${ }^{1, a}$, K.N. Anyfantis ${ }^{1, b}$ \\ ${ }^{1}$ Shipbuilding Technology Laboratory, School of Naval Architecture and Marine Engineering, \\ National Technical University of Athens, Athens, Greece \\ atsouv@mail.ntua.gr, ${ }^{\mathrm{b}} \mathrm{kanyf} @$ central.ntua.gr
}

Keywords: Adhesive bonding, Cohesive laws, Interface elements, Mixed-mode fracture

\begin{abstract}
The present work involves a numerical modelling of the Embedded Process Zone (EPZ) by utilizing the elastoplastic Mode I and Mode II fracture models for the simulation of plastically deforming adhesive joints. A traction-separation law was developed separately for Mode I and Mode II. For the analysis of the mixed-mode fracture processes, the cohesive zones in Mode I and Mode II fracture were assumed uncoupled. The experimental programme involved the fabrication and testing of Double Strap Joints (DSJs) and Single Lap Joints (SLJs). By fitting the numerical results to the experimental ones, the basic cohesive parameters of the problem were defined.
\end{abstract}

\section{Introduction}

Adhesive bonding is gaining more and more interest due to the increasing demand for joining dissimilar structural components, mostly within the framework of designing lightweight structures. During loading, the adhesive material at the crack tip experiences extreme deformation often leading to the development of extensive plasticity and microscopic cracks. This area of the adhesive is denoted as the process zone. When using ductile adhesives, this zone usually extends in a large area in front of the crack tip. During crack growth, two new surfaces are created. Before the formation of the physical crack, these two new surfaces are held together by traction within a cohesive zone. This traction is a function of the relative displacement of the surfaces which is described by a cohesive law. A cohesive law, denoted also as traction-separation law, can be defined for both Mode I and Mode II fractures [1]. Elastic-plastic fracture mechanics have been used to describe adhesive joints with the use of cohesive laws [2,3]. These laws are mostly associated with interface finite elements for the numerical modelling of the process zone. For the analysis of plastically deforming adhesive joints, the Embedded Process Zone (EPZ) approach has been proposed [4]. For applying the EPZ approach, either a coupled or an uncoupled cohesive zone modelling may be utilized [5]. A coupled constitutive relation assumes that both shear and peel tractions depend on both the peel and shear deformation of the bi-material interface. This leads to a mode dependency of the fracture behaviour. The coupled approach is based on the definition of a coupled relationship between Modes I and II [4] that does not yield the same fracture energy for all mode mixtures [5]. On the other hand, according to the uncoupled approach, the traction-separation relationships in Modes I and II are assumed to be uncoupled under mixed mode loading cases [6]. The energy release rate, $\mathrm{J}$, in Modes I and II is defined as the area under the corresponding cohesive law.

The objective of this study is the development of a robust yet flexible cohesive law that can be used to simulate the constitutive behaviour of an adhesive layer under mixed mode loading. The traction-separation parameters are defined separately for Mode I and Mode II, by applying the Jintegral approach in combination with the adhesive material properties. The defined cohesive laws are incorporated into interface finite elements used for modelling the adhesive behaviour of the tested single lap and double strap joints. 


\section{Experimental Procedure}

All substrates (inner adherents and strap adherents) were made of normal marine grade steel, for both SLJ and DSJ configurations. The adhesive used to bond the specimens was Araldite 2015, a relatively stiff two-component epoxy adhesive manufactured by Huntsman Container Corporation Ltd. The geometry of the specimens is given in Fig. 1, showing a side view of them. The width of all specimens was equal to $25 \mathrm{~mm}$. The thickness of the adhesive layer, $t_{a}$, was $0.5 \mathrm{~mm}$ and provisions had been taken during manufacturing to keep this value constant for all specimens. Before bonding the straps of the DSJs, the two inner $10 \mathrm{~mm}$ adherents were placed in contact, without any adhesive in-between them. As for the surface treatment, the common in the shipbuilding industry Sa 2 $\frac{1}{2}$ near white grit blast cleaning was applied (approx. $2.5 \mathrm{~mm}$ grit size) to the bonding surfaces of all substrates. This procedure gave a measured average roughness $(R a)$ of $4.15 \mu \mathrm{m}$. More details can be found in [7]. After the assembly, the specimens were cured in an oven under a uniform pressure loading. According to the adhesive material manufacturer, curing procedure consisted of heating the specimens in $60^{\circ} \mathrm{C}$ for 4 hours, followed by a slow cooling to ambient temperature. The specimens were left in ambient temperature for 48 hours before performing the tests. Specimens were loaded by a uniaxial static tensile displacement, applied with a speed of $0.1 \mathrm{~mm} / \mathrm{min}$ by an MTS hydraulic testing machine, equipped with a $100 \mathrm{kN}$ load cell. During loading the applied displacement together with the reaction forces were monitored.

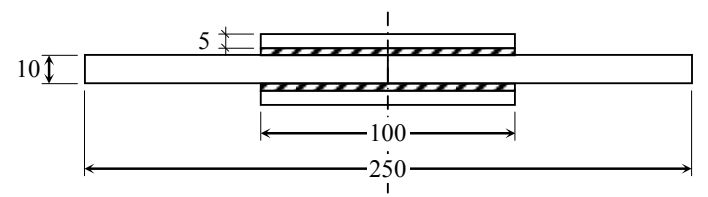

(a)

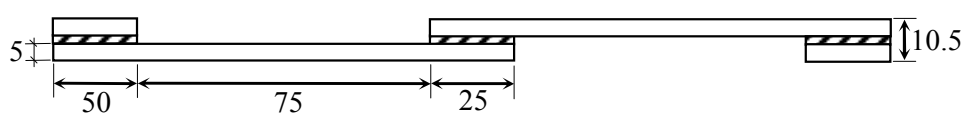

(b)

Fig. 1: Geometry and dimensions of the examined DSJ (a) and SLJ (b)

\section{Interface Element Formulation}

A 6-node interface element has been developed (see Fig. 2) based on the formulation proposed in [1]. The element has two translational degrees of freedom per node, one in each direction of a Cartesian 2D plane. The $12 \times 12$ element tangent stiffness matrix $K$ and the $12 \times 1$ internal force vector $f_{I}$, which are required for the Newton-Raphson nonlinear solution algorithm, are given in a local curvilinear coordinate system by Eq. 1 and Eq. 2, respectively:

$$
\begin{aligned}
& K=b \int_{-1}^{1} B^{T} R \bar{S} B \operatorname{det} J d \xi \\
& f_{I}=b \int_{-1}^{1} B^{T} R \bar{t} \operatorname{det} J d \xi
\end{aligned}
$$

where $b$ denotes the element width in the out-of-plane z-direction. Matrix $B$ contains the quadratic shape functions (Hermitian polynomials) $N(\xi)$, which relate the vector containing the nodal displacements $u$ with the relative displacements of the elements $\bar{\delta}$, multiplied with the rotational matrix $R$. The relative displacements are calculated as the difference between the displacements of the upper $(4,6,5)$ and lower $(1,3,2)$ nodes and are given by:

$$
\bar{\delta}=R^{T} N u \text {. }
$$

Accounting for geometrical non-linearities, a rotational matrix $R$ containing the direction cosines can be defined as 


$$
R=\frac{1}{\operatorname{det} J}\left[\begin{array}{cc}
r_{1} & -r_{2} \\
r_{2} & r_{1}
\end{array}\right] \text { where } \operatorname{det} J=\sqrt{r_{1}^{2}+r_{2}^{2}}
$$

Vector $\bar{t}$ contains the shear and normal tractions $\left\{\bar{t}_{s} \bar{t}_{n}\right\}$ in the local coordinate system as described by the respective cohesive laws. A cohesive law is interpreted as the constitutive equation of the interface to be modelled, given as the function between tractions and relative displacements.

$$
\left\{\begin{array}{ll}
\bar{t}_{s} & \bar{t}_{n}
\end{array}\right\}=F\left(\begin{array}{ll}
\bar{\delta}_{s} & \bar{\delta}_{n}
\end{array}\right) .
$$

Thus, the material stiffness matrix, $\bar{S}$, can be defined by:

$$
\bar{S}=\frac{\partial \bar{t}}{\partial \bar{\delta}}, \quad \text { in matrix form } \quad \bar{S}=\left[\begin{array}{cc}
\bar{S}_{s} & \bar{S}_{s n} \\
\bar{S}_{n s} & \bar{S}_{n}
\end{array}\right]
$$

where $\bar{S}_{s}$ and $\bar{S}_{n}$ denote the derivatives of the interfacial shear and normal tractions in terms of the sliding and normal separation, respectively.

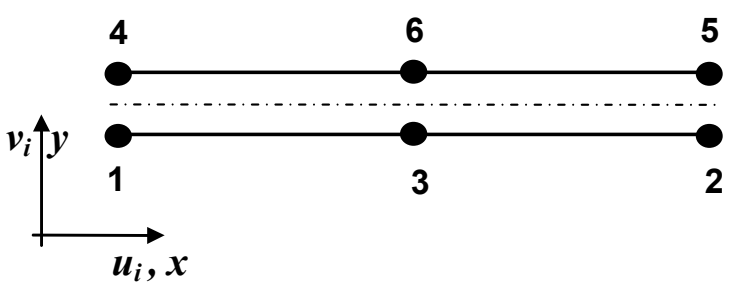

(a)

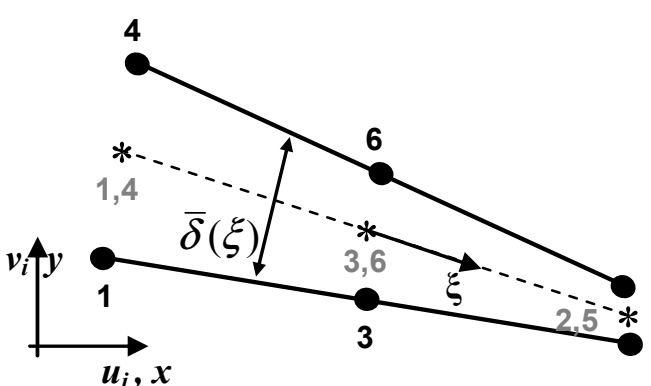

(b)

Fig. 2: 6-node 2D interface finite element in undeformed (a) and deformed state (b)

\section{Finite Element Modelling}

The finite element mesh of the SLJ and DSJ configuration are presented in Fig. 3a and Fig.3b, respectively, together with loading and boundary conditions. Because of its symmetry, only one quarter of the DSJ has been modelled and the respective symmetry boundary conditions have been applied. In order to account for geometrical and material nonlinearities, the Newton-Raphson method has been utilized together with a line search algorithm. A displacement controlled approach is utilized for aiding the convergence of the non-linear solution and avoiding numerical instability issues involved in crack growth analyses, where softening behaviour is apparent. The substrates involved in both types of joints have been modelled with CPS8 continuum 2D elements available in the element library of ABAQUS ${ }^{\circledR} 6.8$. The width of the FE models is $25 \mathrm{~mm}$ in both cases. The elastic material properties of the substrates are $E=210 \mathrm{MPa}$ and $v=0.3$. Since all substrates are very thick with respect to the adhesive layer thickness in the present study, it can be stated with confidence that there will be no yielding of the metal substrates prior to failure of the adhesive and the consequent total failure of the joint [7]. Thus no plasticity properties of steel are needed. Experimental observations have shown that crack initiated and propagated in the area of the adhesive layer, either characterized by adhesive or cohesive failure [7]. In a numerical analysis, an adhesive layer constrained between two continua can be modelled by embedding the process zone in a cohesive law that describes the constitutive behaviour of this layer. Thus, the traditionally used continuum elements have been replaced with interface elements placed between the substrates of the SLJ and DSJ, as shown in Fig. 3a and Fig. 3b, respectively. 


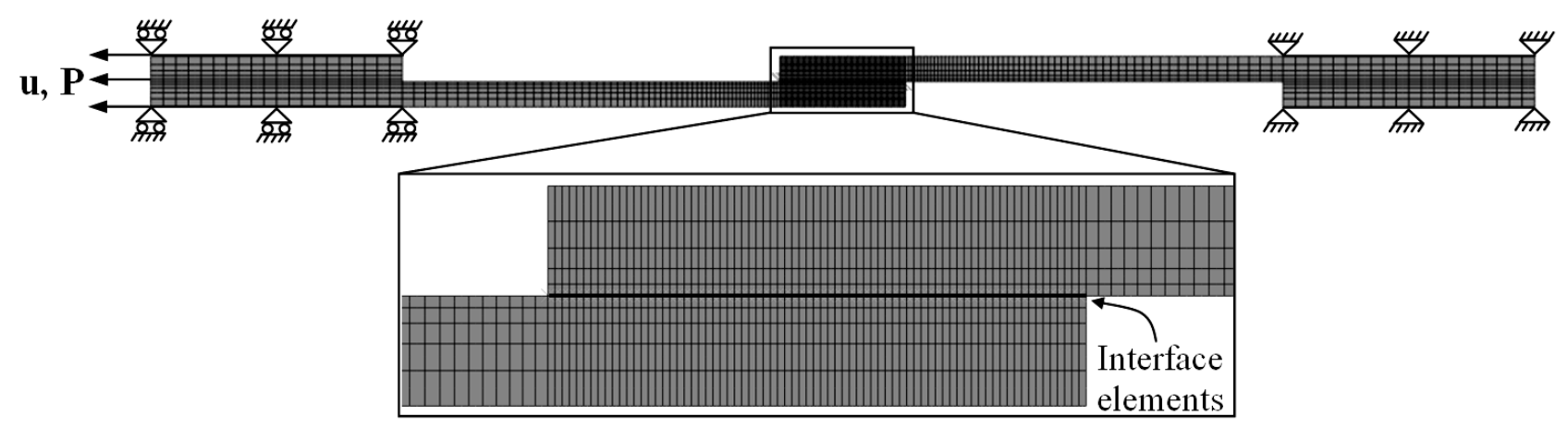

(a)

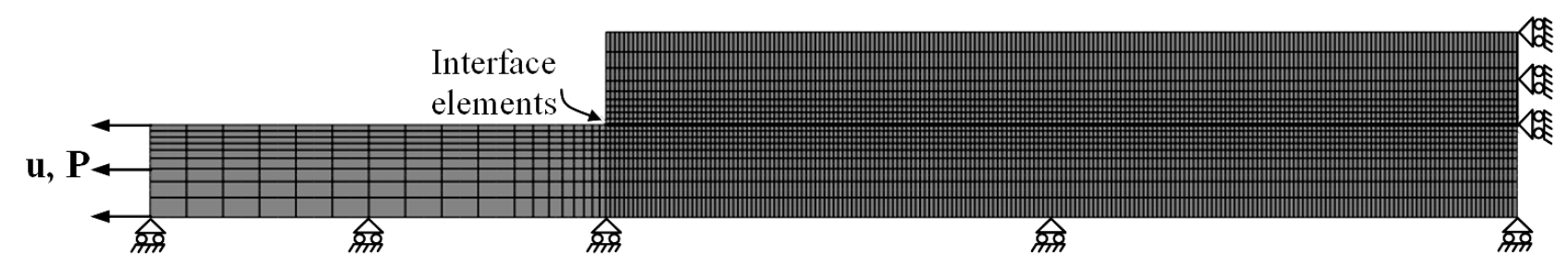

(b)

Fig. 3: Mesh, boundary and loading conditions of the SLJ (a) and DSJ (b)

For the development of cohesive laws that describe the behaviour of an adhesive layer, three sets of characteristic constitutive parameters must be defined, separately for Mode I and II [5]. The first set involves the initial elastic response of the adhesive and is described by the ratio between the Young's or shear modulus and the adhesive thickness $E_{a} / t_{a}$ and $G_{a} / t_{a}$, for Mode I and Mode II respectively. In the present study, $E_{a}=2 \mathrm{GPa}$ is the Young's modulus and $G_{a}=0.77 \mathrm{GPa}$ is the shear modulus of the bulk adhesive [8]. The rest two sets of cohesive parameters involve the maximum attained stress $\left(\sigma_{0}\right.$ and $\left.\tau_{0}\right)$ together with their respective relative displacement values $\left(\delta_{I, 0}\right.$, $\left.\delta_{I I, 0}\right)$ and the maximum separations after which tractions vanish $\left(\delta_{I, C}, \delta_{I I, C}\right)$. Having defined the cohesive parameters, the total energy uptake can be calculated for Mode I $\left(J_{I C}\right)$ and Mode II $\left(J_{I I C}\right)$ respectively as [9]:

$$
J_{I C}=\int_{0}^{\delta_{I, C}} \sigma d \delta_{I} \quad \& \quad J_{I I C}=\int_{0}^{\delta_{I I, C}} \tau d \delta_{I I} .
$$

These parameters evolve together as a natural result of the interaction between the deformation of the substrates and the behaviour of the two separate cohesive laws. Thus, the total energy release rate absorbed during mixed mode loading is given by the summation of the fracture toughness of the pure cohesive laws in the following form: $J=J_{I}+J_{I I}$.

Apart from the aforementioned sets of cohesive parameters, the well known cohesive behaviour (traction-separation function) has to be defined. In this study, an exponential function has been assumed to describe the ductile adhesive behaviour in both Modes I and II up to the point of maximum stress, followed by a linear softening behaviour after this point. The selection of the exponential function was based on the similar behaviour of the stress-strain response of the adhesive. The cohesive laws that describe the EPZ of the ductile adhesive layer used in this study are presented in Fig. 4. From the cohesive parameters shown in this figure, some magnitudes $\left(\sigma_{0}\right.$, $\delta_{I, 0}$ and $\left.\delta_{I I, C}\right)$ were taken from data available in the literature $[9,10]$, whereas the others $\left(\tau_{0}, \delta_{I, C}\right.$ and $\left.\delta_{I I, 0}\right)$ have been determined by an inverse method, i.e. by fitting the numerically calculated loaddisplacement response of the SLJ and DSJ configuration over the respective experimentally measured data. 


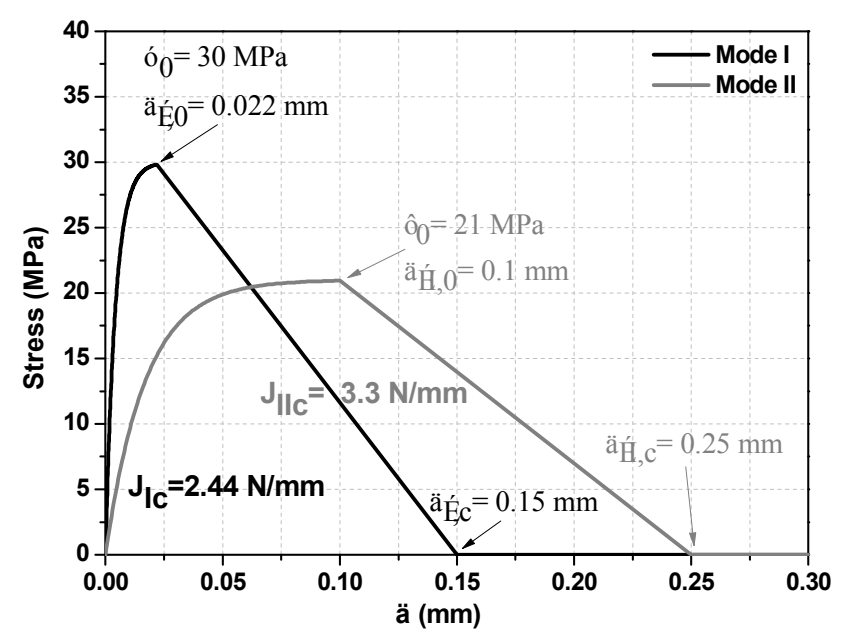

(a)

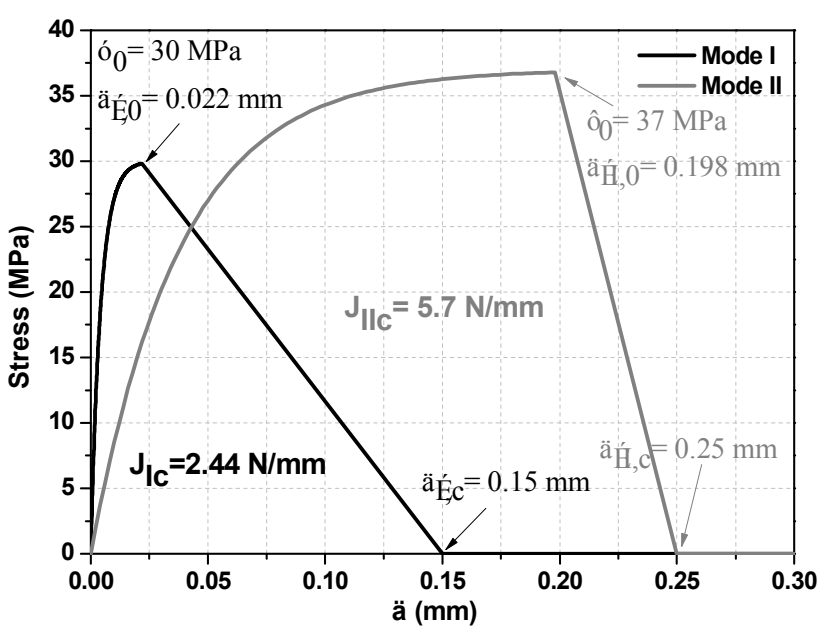

(b)

Fig. 4: Exponential cohesive laws with linear softening used for SLJ (a) and DSJ (b)

\section{Numerical and experimental results}

Fig. 5 illustrates a comparison between the load/displacement curves obtained from the SLJ and DSJ tests and the Finite Element Analysis (FEA) results based on the cohesive laws shown in Fig. 4. Both experimental curves obtained from SLJ and DSJ tests denote an initially linear response, followed by increasing non-linearities as the adhesive layer begins to substantially deform plastically. Fig. 5 shows that, although the numerical results were fitted to the experimental data by selecting appropriate values for some of the cohesive parameters $\left(\tau_{0}, \delta_{I, C}\right.$ and $\left.\delta_{I I, 0}\right)$, the proposed exponential character of the cohesive law proved adequate for modelling the elastoplastic behaviour of the ductile adhesive up to the maximum attained stress in the opening Mode I and the sliding Mode II responses. The DSJ attained much higher loads than the SLJ. This is reasonable, since the bonding surface of the DSJ is four times larger than that of the SLJ. Additionally, due to the symmetry of the DSJ configuration, the adhesive layer is stressed mostly in shear whereas the geometrical eccentricity of the SLJ leads to the development of cleavage stresses at the adhesive edge. Cleavage stresses are the most critical stresses that jeopardize the integrity of the joint by leading to premature failure. According to experimental observations and numerical predictions, in both cases and after the maximum attained load, the joints debonded in a sudden manner until complete failure. This debonding procedure is represented by the softening behaviour of the load/displacement curves after the maximum load. The sudden debonding behaviour was simulated with the use of a linear softening function which was incorporated separately in each of the cohesive laws describing pure mode failure. The FEA of the DSJ additionally captured the softening behaviour, as depicted in Fig. 5b. The SLJ experimental curve exhibits a softening response characterized by a slower rate in comparison to the rate of the respective DSJ softening behaviour. In this case, the cohesive softening law used for the simulation of the EPZ of the SLJ (see Fig. 4a) in combination with the uncoupled approach, did not capture the softening behaviour of the experimentally obtained response curve, as shown in Fig. 5a. Nevertheless it is essential to state that, instead of the shear stresses, the cleavage ones being developed at the adhesive edge are responsible for crack initiation, as cleavage stresses peak at $0.022 \mathrm{~mm}$ with a value of $30 \mathrm{MPa}$, whereas shear stresses peak at $0.1 \mathrm{~mm}$ having a value of $21 \mathrm{MPa}$. After crack initiation, total failure of the adhesive depends on the developed shear cohesive law. On the other hand, although the DSJ configuration is a shear lap type of joint in which the adhesive is loaded mostly in pure shear, the Mode I failure process must be additionaly incorporated into the mixed mode cohesive law in order to numerically capture failure initiation. Finally, the cohesive law in Mode II contributes mostly to the total energy release rate during the mixed mode fracture process of the DSJ, since $J_{I I C}$ is almost twice as $J_{I C}$. 


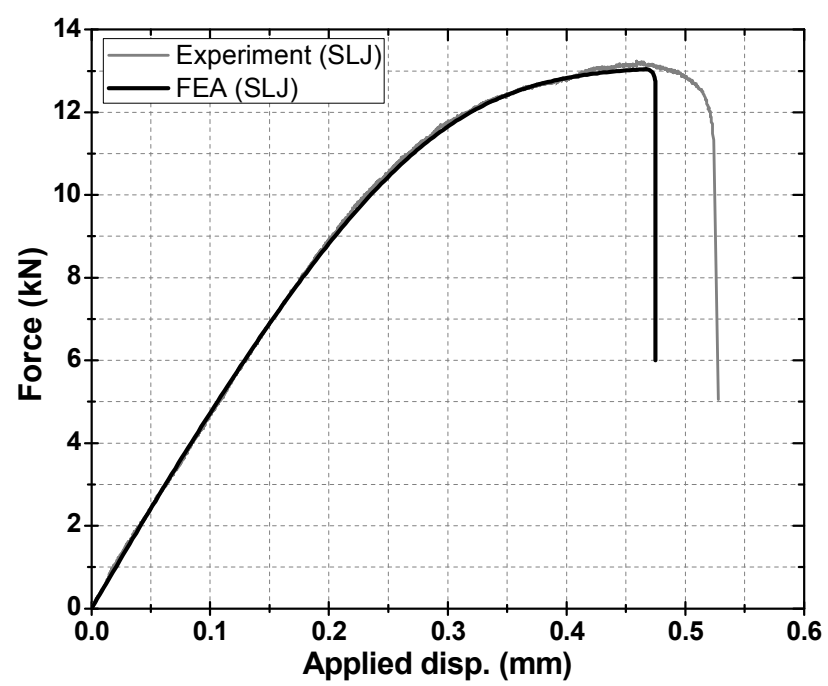

(a)

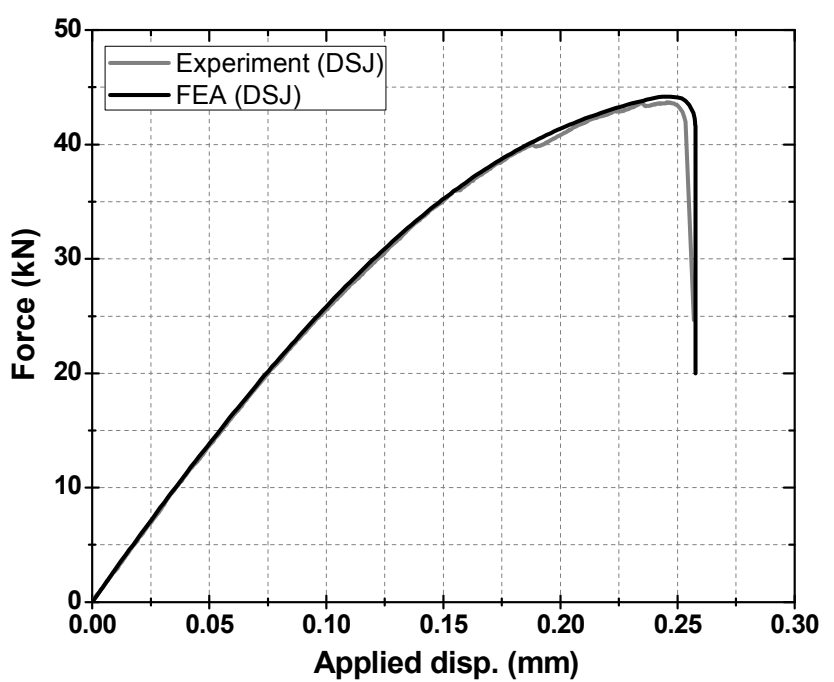

(b)

Fig. 5: Reaction force vs. applied displacement response of SLJ (a) and DSJ (b)

\section{Conclusions}

Two different cohesive laws have been developed to account for the EPZ of a ductile adhesive, one in Mode I and one in Mode II. The developed cohesive laws were used to describe the constitutive relation of user defined interface elements. These elements were utilized to model the behaviour of the adhesive and were implemented in-between the bondline of the substrates, so as to simulate the mixed-mode fracture processes of a SLJ and a DSJ. The cohesive laws were assumed uncoupled. The cohesive parameters were determined implicitly, by fitting the numerical results on the respective experimental data. The excellent agreement between the numerical and the experimental results indicate the validity of the exponential type cohesive law for modelling the elastoplastic behaviour of a ductile adhesive, at least for the present case of the very stiff adhesives where the adhesive behaviour dominates. The differences found in the softening part of the SLJ response owe probably to the linear softening law adopted herein.

\section{References}

[1] V.K. Goyal, E.R. Johnson and V.K. Goyal: Comp. Struct. Vol. 82 (2008), p. 434

[2] R.D.S.G. Campilho, M.F.S.F. de Moura, and J.J.M.S. Domingues: Int. J. Solid. Struct. Vol. 45 (2008), p. 1497

[3] Leffler, K.S. Alfredsson and U.Stigh: Int. J. Solid. Struct. Vol. 44 (2007), p. 530

[4] V. Tvergaard and W.J. Hutchinson: J. Mech. Phys. Solids Vol. 40 (1992), p. 1337

[5] L.J. Hogberg: Int. J. Fract. (2006) Vol. 141, p.549

[6] M.S. Kafkalidis and M.D. Thouless: Int. J. Solid. Struct. Vol. 39 (2002), p. 4367

[7] K.N. Anyfantis and N.G. Tsouvalis, in: Analysis and Design of Marine Structures, edited by G. Soares and P. Das, Proceedings of MARSTRUCT 2009, 2nd International Conference on Marine Structures - Analysis and Design of Marine Structures, Taylor \& Francis Group, London (2009), pp. 387-392

[8] Huntsman data sheet, http://www.intertronics.co.uk/data/ara2015.pdf

[9] Q.D. Yang and M.D. Thouless: Int. J. Fract. (2001) Vol. 175, p.175

[10]M.F.S.F. de Moura, R.D.S.G. Campilho and J.P.M. Concalves: Int. J. Solid. Struct. Vol. 46 (2009), p. 1589 